\title{
Exuberant subcutaneous emphysema after thoracoscopic surgery - case report
}

\section{Duarte S., Sobreira Fernandes D., Saraiva A., Antunes R., Figueiredo D \\ Centro Hospitalar do Porto, Dept of Anaesthesiology \& Intensive Care, Porto, Portugal}

\section{Background}

Subcutaneous emphysema (SE) is usually self-limited, but when it develops in the upper chest and neck may lead to severe upper airway obstruction and fatal respiratory failure. SE is usually related to tracheobronchial injuries (TI) and therefore can be related to intubation as well as cervical or thoracic procedures. Patient characteristics and technical difficulties increase the risk of SE.

\section{Case Report:}

A 62-year-old female, ASA 3, with Behçet disease and obesity was proposed to an elective right thoracoscopic cervico-thoracic sympathectomy due to post-traumatic right arm chronic pain and oedema.

Patient was selectively intubated with a left double lumen $37 \mathrm{Fr}$ endotracheal tube. Intubation was uneventful and confirmed by fibroscopy.

Surgical procedure was laborious and prolonged due to several pleural adhesions and difficult pulmonary deflation. At the end of the surgery chest $X$-ray was normal and patient was safely extubated. The patient was discharged from post-anaesthetic care unit two hours after the end of the procedure.

Six hours later, was admitted in the emergency room for exuberant $\mathrm{SE}$, hoarseness and dyspnoea. A blood gasometry showed a respiratory acidosis with normal oxygenation ( $\mathrm{FiO} 2$ of $50 \% ; \mathrm{pH}$ $7,30, \mathrm{pCO} 257,3 \mathrm{mmHg}$ ). A chest X-ray was performed and a right chest drain was inserted, without clinical resolution. A head to pelvis CT scan was performed showing subcutaneous emphysema from palpebral level to lumbar flanks, with predominance in the cervical level (with extrinsic airway compression due to retropharyngeal emphysema), and thoracic level (with extensive subcutaneous emphysema and mediastinal emphysema, without significant pneumothorax) (image 1). Bronchoscopy only showed tracheal haemorrhagic suffusions immediately after vocal cords without any visible leak.

Patient was maintained in spontaneous ventilation with high concentration $\mathrm{O}_{2}$ flow and careful monitored in intensive care unit. Four days after was transferred to surgery ward and in the eight postoperative day was discharged, without complains
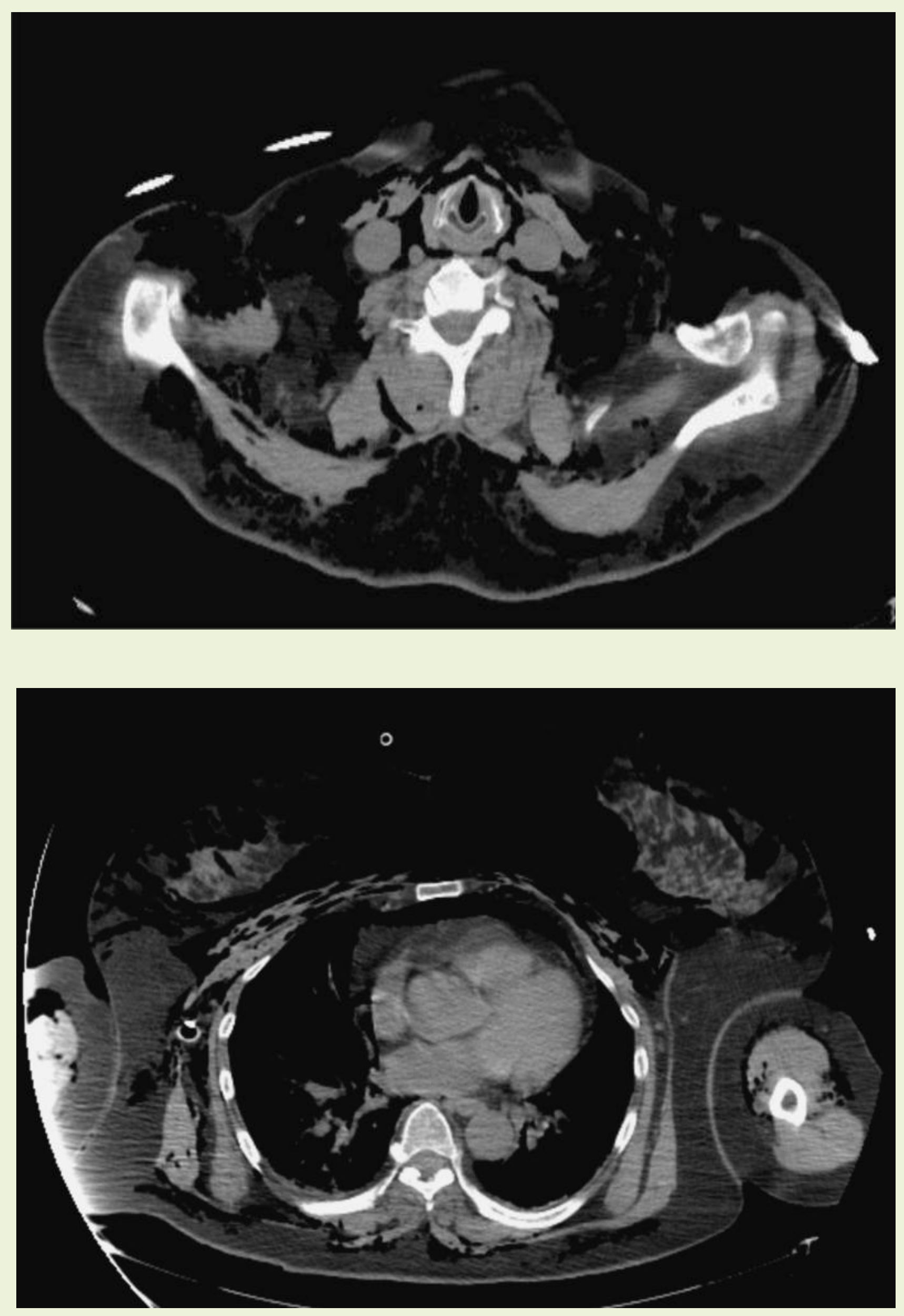

\section{Discussion}

Anaesthetic and surgical technical difficulties and Behçet disease increase the risk of $\mathrm{Tl}^{2}$. SE is the most common clinical finding in $\mathrm{TI}$ and usually presents several hours after surgery. The incidence of iatrogenic $\mathrm{Tl}$ after elective orotracheal intubations is rare. Bronchoscopy is the best method to confirm a TI.

In this case, the SE appears to be related to a small TI in distal airway, caused by technical difficulties during the procedure due to several pleural adhesions.

Conservative treatment is recommended in patients with mild symptoms, small ruptures with no air leakage in spontaneous ventilating patients.

\section{Learning points}

Surgical, anaesthetic and patient risk factors for postoperative SE; SE differential diagnosis; Airway management of the patient with extensive facial and neck SE; Management of the patient with extensive SE; Treatment options of the patient with SE. 